\title{
Optimal and Fast Denoising of AWGN using Cluster based and Filtering Approach
}

\author{
Mayuri D. Kulkarni \\ $2^{\text {nd }}$ Year M.Tech (Student) \\ NIIST,Bhopal \\ RGPV, Bhopal
}

\author{
Sitendra Tamrakar \\ Assistant Professor \\ NIIST, Bhopal \\ RGPV, Bhopal
}

\author{
Shatendra Dubey \\ Assistant Professor \\ NIIST, Bhopal \\ RGPV, Bhopal
}

\begin{abstract}
This paper presents the novel framework, which provides the denoising of image which is corrupted because of additive white Gaussian Noise. This introduction of noise causes degradation of an image. In proposed framework, the image is preprocessed after preprocessing, similar clusters of are accumulated for the filtering. After getting similar clusters, Adaptive Wiener filtering is used to denoise an image. The image quality assessment factors used are Peak Signal-tonoise ratio (PSNR) and structural similarity index (SSIM).
\end{abstract}

\section{Keywords}

Denoising,AWGN,K-means, Adaptive Wiener filter

\section{INTRODUCTION}

In recent era, applicability of images is almost in all the fields. These fields are remote sensing, technical diagnostics, autonomous vehicle guidance, biomedical imaging and automatic surveillance.Those images are used by the researches, doctors, scientists etc. Cameras areused to capture the images. This camera, uses the CCD or C-MOS .The CCD or because of C-MOS which introduces the noise in an image.

Noise is an unwanted signal which introduces in an image. This unwanted signal degrades the quality of an image. Image carries a lot of information such as clarity, brightness, sharpness, curves etc. This information degrades because of unwanted signal introduction. This information is useful for analysis, recognition and segmentation.

Noise is introduced by the sensor noise causes by poor luminance.These sensors measure the charge induced by incident photons on mesh of electro-optical elements [1].

Gaussian noise is evenly distributed over the signal. Each pixel in noisy image is the sum of true pixel value and a random Gaussian distributed noise value.The Gaussian function can be considered as one of the considered as one of the classical point spread function as

$$
h(z)=\frac{1}{\sqrt{2 \pi \sigma}} e^{-(z-\mu)^{2}} /-2 \sigma^{2} \ldots \ldots(1)
$$

where $\mathrm{z}$ represents the grey level, $\mu$ mean value and $\sigma$ standard deviation.

Additive noise mode adds the unwanted signal in an original image which cause the degradation of an image which is shown in following function

$$
u_{0}(x, y)=f(x, y)+n(x, y) .
$$

where $\mathrm{f}$ is ideal image, $u_{0}$ is noised image, $\mathrm{n}$ denotes the additive noise with zero mean and variance.

In case of Additive White Gaussian Noise, the original image $f(x, y)$ is degraded with $n(x, y)$ from eq. (2) which is nothing but the Gaussian function in eq. (1). In case of AWGN, the mean $\mu$ is set to zero.

PSNR generally indicates that reconstruction of higher quality image, which the ratio between the maximum possible power of a signal and the power of corrupting noise that affects the fidelity of its representation as in eq.(3)

$$
P S N R=10 \log _{10} \frac{255^{2}}{M S E}
$$

where MSE $=\frac{1}{m n} \sum_{i=0}^{m-1} \sum_{j=0}^{n-1}[I(i, j)-k(i, j)]^{2}$ where $\mathrm{I}(\mathrm{i}, \mathrm{j})$ is noisy image and $\mathrm{k}(\mathrm{i}, \mathrm{j})$ is filtered image.

Wang et al. proposed a new quality assessment method based on the structural information of two images, has proved the good measures for different kinds of images through SSIM. It uses three levels of comparison which are luminance, contrast and structural. SSIM is calculated as follows [2] which is shown in eq. (4):

$$
\operatorname{SSIM}(x, y)=\frac{\left(2 \mu_{x} \mu_{y}+c_{1}\right)\left(2 \sigma_{x y}+c_{2}\right)}{\left(\mu_{x}^{2}+\mu_{y}^{2}+c_{1}\right)\left(\sigma_{x}^{2}+\sigma_{y}^{2}+c_{2}\right)} \ldots \ldots
$$

where $\mu_{x}$ the average of $\mathrm{x}, \mu_{y}$ the average of $\mathrm{y}, \sigma_{x}^{2}$ the variance of $\mathrm{x}, \sigma_{y}^{2}$ the variance of $\mathrm{y}$,

$\sigma_{x y}$ The covariance of $x$ and $y, c_{1}=(k 1 L)^{2}, c_{2}=(k 2 L)^{2}$ two variables to stabilize the division with weak denominator $\mathrm{k} 1=0.01 \& \mathrm{k} 2=0.03$ by default

This paper contains literature survey which describes various approaches suggested for AWGN denoising. And work did in this field. Further it contains the proposed framework which is provides the optimal results for AWGN denoising. Paper ends with results and discussions with conclusion.

\section{LITERARTURE REVIEW}

While denoising an image, estimation of noise present in an image is very essential. Estimation of noise in an image is estimated by considering approaches as filtering, block or transformed-based approach [10]

Tuning parameter in denoising is adjusted in case of collaborative approach. Collaborative approach uses the filtering to the homogeneous groups[11]. 
To recover images from the Gaussian additive white noise is really challenging issue. When the denoising technique is used, it affects the quality of the image, which causes the loss of information in an image at the edges, sharpness so that the visual quality of the image is degraded. Patch based approach uses similar patches to remove noise from the patch using various filtering techniques [3] [4] [5].

Patch-Based Near-Optimal Image Denoising filter statistically motivated by the statistical analysis performance for the Gaussian additive white noise .In this framework, locally optimal wiener filter is developed where parameters as weight and covariance are learned from the both geometrically and photometrical similarity [3].

To identify geometrically and photometrical similar patches are identified for each reference, estimated parameters of reference patches to denoise image from most similar patches weights are computed [4] [5].

Unsupervised method that used no library for image patches and smoothing works in joint spatial-range domain as nonlocal mean filter as smoothing window .In proposing method maximum likelihood of patches are estimates as its mean and variance along that patch size is estimated so that optimum window is selection is possible which will give the optimal result [5].

Segmentation algorithms can be classified into different categories as feature's threshold, region based and clustering [7]. A portion of an image which wanted to be smooth, estimates the noise levels automatically using segmentation approach [7] [8].

Clustering is an unsupervised classification designed to group a set of data sample with similar characteristics into larger units of clusters [9]. Cluster analysis is used to analysis the "similarity" between objects and "dissimilarity" to the objects belongs to another cluster. In K-means algorithm, predefined numbers of clusters are initialized and centroids predefined randomly. The dimensions of each centroid are same as the cluster based on closeness which is determined by the Euclidian distance measures [9] [7].

\section{PROPOSED FRAMEWORK}

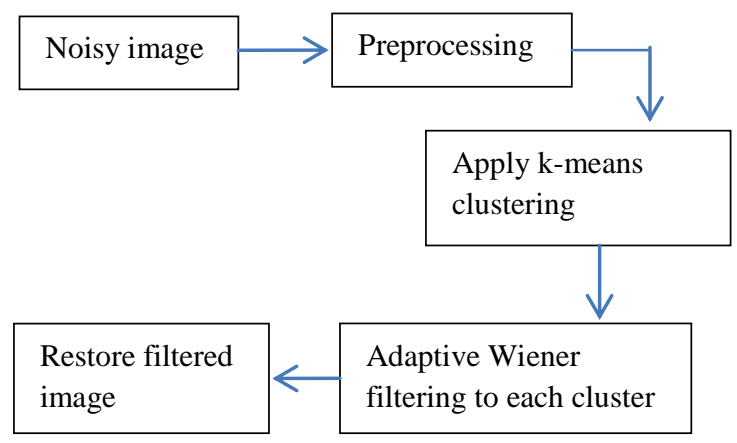

\section{Fig 1 : Proposed framework}

In our proposed method shown in fig 1, proposed method uses the input as the natural imaging set which is corrupted by the Gaussian additive white noise with the various levels of variance.

This noisy image is preprocessed using order static filter \& median filter. This preprocessing is used for the preservation of high frequency signals as well as low frequency signal.
High frequency signals are preserved using order static filters whereas median filtering is used for the smoothing purpose.

After preprocessing similar mean clusters formed using kmeans clustering technique. K-means uses Euclidean distance technique for centroid calculation.K-mean provides the similar clusters which are then used for the filtering purpose. Adaptive Wiener filter is used to remove noise from each cluster. These optimal denoising steps are given below :

1. Input original image from natural image set.

2. Add noise in image with level of variance either Gaussian additive white noise.

3. Apply prefiltering process to the noisy image

a. Preprocess the image using $2 \mathrm{D}$ order statistic filter

b. Apply the median filter

c. Perform correlation operation on an image.

4. After getting preprocessed apply k-mean to the preprocessed image by specifying the number of clusters to be formed in the image.

5. Apply adaptive Wiener filter to the similar cluster.

6. Combine all the clustered images to form the complete denoise image.

7. Calculate the mean, Peak-Signal-Noise Ratio and Structural Similarity Index.

\section{RESULT}

Here, compared the proposed denoising method on various images at different level of noise. And results are shown for different Levels of Gaussian additive white noise shown in fig 2.

Table. 1 shows quality assessment parameters as PSNR, SSIM for different level of noise variance.

Proposed framework is compared with the previous method and which provides the improved result for noise level more than 5. This comparative analysis is Also graphical analysis of the proposed method with the previous methods is shown in fig 3 .

This framework uses performance improvement functions to improve the speed of operation of preprocessing. Those improved functions are as ordfilt 2 , medfilt 2 , imfilter. The completion time to denoise a grey scale image of size $256 \times 256$ is $15 \mathrm{Sec}$ and for $512 \times 512$ is up to50 sec. This performance is analyzed on an Intel i3 processor with MATLAB version 2010a.

In terms of computational complexity, the proposed method provides the optimum results than that of the previous method [3].

Table 2 shows the comparative analysis of time computation of previous methods over proposed method. 

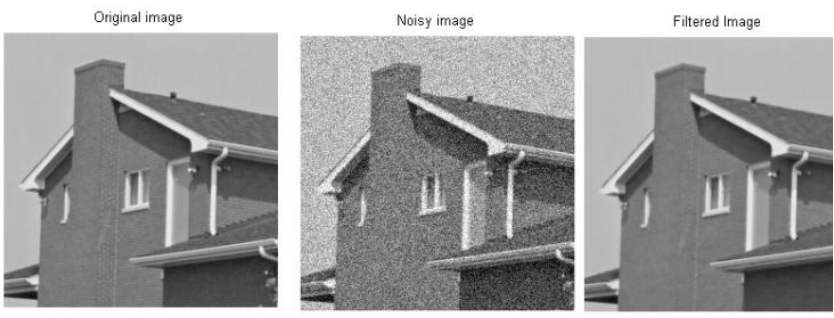

Fig 1 : Original image, Noise as Gaussian additive white noise with sigma 25 (Noisy image), Final filtered image.

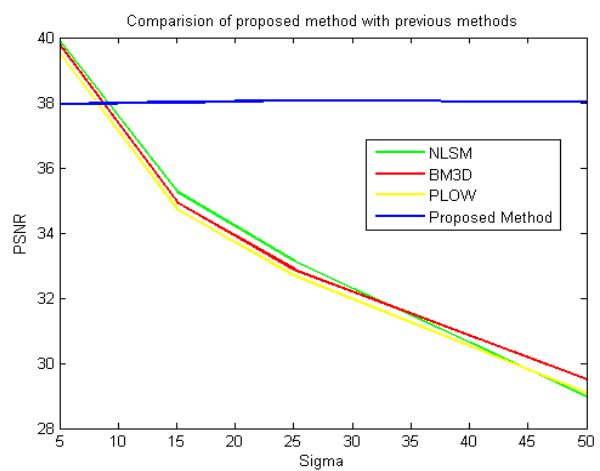

Fig 3 : Graphical Analysis of Proposed Method with NLSM, BM3D, PLOW [3]

Table I. Results of proposed framework, for Gaussian Additive White noise for various levels of noise variance and for the images as House, Lena, Barbara, Boat and

Pepper are assessed on the PSNR(Upper) and $\operatorname{SSIM(Lower).~}$

\begin{tabular}{|l|l|l|l|l|l|}
\hline $\begin{array}{l}\text { Noise } \\
\text { variance }\end{array}$ & House & Lena & Barbara & Boat & Pepper \\
\hline \multirow{2}{*}{$\mathbf{2}$} & 38.03 & 36.12 & 38.15 & 36.79 & 38.77 \\
& 0.906 & 0.938 & 0.972 & 0.934 & 0.959 \\
\hline \multirow{2}{*}{$\mathbf{0}$} & 37.97 & 36.12 & 38.19 & 36.78 & 38.74 \\
& 0.905 & 0.939 & 0.973 & 0.933 & 0.958 \\
\hline \multirow{2}{*}{$\mathbf{5}$} & 38.005 & 36.08 & 38.17 & 36.81 & 38.80 \\
& 0.906 & 0.938 & 0.973 & 0.934 & 0.959 \\
\hline \multirow{2}{*}{$\mathbf{2 0}$} & 37.97 & 36.09 & 38.18 & 36.79 & 38.75 \\
& 0.904 & 0.938 & 0.973 & 0.933 & 0.958 \\
\hline \multirow{2}{*}{$\mathbf{5}$} & 37.99 & 36.10 & 38.17 & 36.81 & 38.81 \\
& 0.905 & 0.938 & 0.972 & 0.934 & 0.958 \\
\hline \multirow{2}{*}{$\mathbf{5 0}$} & 38.034 & 36.11 & 38.20 & 36.79 & 38.79 \\
& 0.905 & 0.938 & 0.973 & 0.933 & 0.958 \\
\hline
\end{tabular}

Table 2. Time Computation for denoising gray image of size 256x256

\begin{tabular}{|l|l|}
\hline Method & Time Taken for Denoising \\
\hline PLOW & $75 \mathrm{sec}$ \\
\hline NLSM & $170 \mathrm{Sec}$ \\
\hline BM3D & $17 \mathrm{Sec}$ (minor drop of 0.2 dB in PSNR) \\
\hline $\begin{array}{l}\text { Proposed } \\
\text { Method }\end{array}$ & $15 \mathrm{sec}$ \\
\hline
\end{tabular}

\section{CONCLUSION}

In the propose framework, uses the collaborative approach for the noise estimation. Parameter for noise estimation is decided by the filters. As clustering technique k-means is used to collect homogeneous elements in an image .This homogeneous elements in an image is filtered with Adaptive Wiener filter. This results into optimal denoising technique. This framework can be extended for color images which will be denoise in minimum time.

\section{REFERENCES}

[1] Vladimir Petro Vic ,Costas S. Xydeas, " Sensor noise effects on signal-level image fusion performance" , Information fusion 4 (2003),167-183

[2] Santiago Aja-Fern Andez, Ra Ul San Jos E Est Epar, Carlos Alberola-L Opez And Carl-Fredrik Westin , “' Image Quality Assessment Based On Local Variance”, Proceedings Of The 28th IEEE EMBS Annual International Conference New York City, USA, Aug 30Sept 3, 2006

[3] Priyam Chatterjee And Peyman Milanfar, "Patch-Based Near-Optimal Image Denoising", IEEE Transactions On Image Processing, April 2012, Vol. 21, No. 4

[4] Priyam Chatterjee And Peyman Milanfar," Patch-Based Locally Optimal Denoising” , $2011,18^{\text {th }}$ IEEE International Conference On Image Processing, 978-14577-1302- 6/11,2011

[5] Charles Kervrann And Jérôme Boulanger , "Optimal Spatial Adaptation For Patch-Based Image Denoising", IEEE Transactions On Image Processing, Vol. 15, No. 10, October 2006

[6] Ioannis Pits And Anastasios Venetsanopoulos , "Order Statistics in Digital Image Processing ", Proceedings of IEEE, Vol ,80, No. 12 ,December 1992.

[7] Siti Noraini Sulaiman, Nor Ashidi Mat Isa, "DenoisingBased Clustering Algorithms For Segmentation Of Low Level Salt-And-Pepper Noise-Corrupted Images" , IEEE Transactions On Consumer Electronics, Vol. 56, No. 4, November 2010

[8] Ce Liu, Richard Szeliski,Sing Bing Kang,C. Lawrence Zitnick,William T. Freeman, "Automatic Estimation And Removal Of Noise From A Single Image", IEEE Transactions On Pattern Analysis And Machine Intelligence Vol. 30, No. 2, February 2008

[9] S. Thilagamani , N. Shanthi, " A Survey On Image Segmentation Through Clustering", International Journal Of Research And Reviews In Information Sciences Vol. 1, No. 1, March 2011

[10] Mayuri D. Kulkarni , Sitendra Tamrakar ,Shatendra Dubey , A Preliminary study of denoising technique", Elixir Comp. Sci. \& Engg. 67 (2014) 21442-21444 ,ISSN :2229-712X

[11] Ce Liu, Richard Szeliski, Sing Bing Kang,C. Lawrence Zitnick, and William T. Freeman, "Automatic Estimation and Removal ofNoise from a Single Image" , IEEE Transactions On Pattern Analysis And Machine Intelligence, Vol. 30, No. 2, February 2008 